This is the peer reviewed version of the following article: Blank, H., Diedenhofen, B. and Musch, J. (2015),

Looking back on the London Olympics: Independent outcome and hindsight effects in decision evaluation.

British Journal of Social Psychology, 54: 798-807. doi: 10.1111/bjso.12116, which has been published in final

form at

http://onlinelibrary.wilev.com/doi/10.1111/biso.12116/abstract?userlsAuthenticated=false\&deniedAccessCustom isedMessage $=$

This article may be used for non-commercial purposes in accordance with Wiley Terms and Conditions for SelfArchiving.

This current archived file is the accepted version of the paper and also includes all corrections made at the journal article proofing stage. Page breaks corresponding to the published version are provided.

\title{
Looking back on the London Olympics: Independent outcome and hindsight effects in decision evaluation
}

\author{
Hartmut Blank $^{1}$, Birk Diedenhofen ${ }^{2}$ and Jochen Musch ${ }^{2}$ \\ ${ }^{1}$ University of Portsmouth, UK \\ ${ }^{2}$ University of Düsseldorf, Germany
}

Correspondence should be addressed to Hartmut Blank, Department of Psychology, University of Portsmouth, King Henry Building, King Henry I Street, Portsmouth, Hampshire, UK (email: hartmut.blank@ port.ac.uk).

\begin{abstract}
Outcome bias and hindsight bias are related, but how exactly? To remedy theoretical ambiguity and nonexistent directly relevant empirical research, we contrast an older idea (Baron \& Hershey, 1988, J. Pers. Soc. Psychol., 54, 569) that sees outcome bias as partly mediated through hindsight bias with the idea that the two biases independently affect decision evaluations. In an Internet study of retrospections on the 2012 London Olympics, evaluations of the Games' success and its foreseeability had independent effects on evaluations of the International Olympic Committee's decision to award the Olympics to London; there was no evidence of mediation. Further theoretical discussion emphasizes the need to distinguish between a holistic assessment of decisions and a more specific assessment of the decision-making process in future outcome bias research.
\end{abstract}

How we look back on the past is shaped, very broadly, by how things turned out. This is a hardly disputable fact of life, but within the remit of this general principle scholars have drawn attention to two more specific and problematic phenomena - outcome bias and hindsight bias. In this report, we (1) review and clarify the conceptual relation between outcome bias and hindsight bias (or outcome and hindsight effects, in a less normatively laden language $)^{1}$ and (2) present findings bearing on this relation from a study of retrospections on the 2012 London Olympics. 


\section{Outcome bias and hindsight bias}

Both outcome bias and hindsight bias stem from outcome knowledge, but focus on different effects of such outcome knowledge. In hindsight bias, the effects are cognitive -

\footnotetext{
${ }^{1}$ An issue that features in many discussions of outcome effects (Baron \& Hershey, 1988; Hershey \& Baron, 1992, 1995; Jones, Yurak, \& Frisch, 1997; Mowen \& Stone, 1992; Rachlinski, 1998; Robbennolt, 2000) is to what degree it is normatively appropriate or inappropriate to take outcome information into account when evaluating behaviour. In a nutshell, speaking of a bias is justified to the degree that outcome information is given undue weight, relative to other information about the adequacy of the decision at the time or the behaviour under the circumstances (i.e., before the outcomes materialized). Normative issues have been discussed with respect to hindsight bias as well (Fischhoff, 1975; Hawkins \& Hastie, 1990), the general argument being that we can speak of a bias as soon as people are unaware, or even denying, that outcome feedback has influenced their judgements. As these normative issues are orthogonal to our main concern, the relation between outcome and hindsight bias, we will not explore them more deeply (except briefly in the discussion section), but will often adopt the more neutral terminology of outcome and hindsight effects.
}

[798]

[799]

specifically, overestimations of the foreseeability and inevitability of event outcomes, as well as distorted memories of initial judgements or predictions (see Blank, Musch, \& Pohl, 2007; Hawkins \& Hastie, 1990;

Hoffrage \& Pohl, 2003; Rachlinski, 1998; Roese \& Vohs, 2012, for introductions). By contrast, outcome bias focuses on evaluative effects - biased evaluations of someone's behaviour or performance by associated outcomes (see Baron \& Hershey, 1988; Rachlinski, 1998; Robbennolt, 2000, for introductions and overviews). For example, if a risky operation on a patient resulted in a fatal outcome, hindsight bias would manifest itself in exaggerated perceptions of the foreseeability of the fatal outcome, whereas outcome bias would be seen in negative evaluations of the decision to perform the operation and/or in negative evaluations of the surgeon's competence. The question of interest is whether there is some link between these different perceptions and evaluations.

\section{The relation between outcome bias and hindsight bias}

Outcome bias and hindsight bias are often not very clearly distinguished in the literature, and therefore their relation remains unclear, too (see Additional Materials for an overview of conceptions). A notable exception is found in a seminal paper by Baron and Hershey (1988), who argue that outcome feedback can influence evaluations either directly or indirectly, through biased perceptions of outcome probabilities (i.e., through hindsight bias, p. 570). Other researchers endorse - less explicitly - the same idea (Brown \& Solomon, 1987; Clarkson, Emby, \& Watt, 2002; Gupta, Schriger, \& Tabas, 2011; Lipshitz \& Barak, 1995; Mazzocco, Alicke, $\&$ Davis, 2004; Rachlinski, 1998). As our own idea of the relation between hindsight bias and outcome bias (see below) starts from this view, we illustrate the logic of direct (outcome bias) and indirect (via hindsight bias) effects of outcome knowledge on evaluations in some detail.

In our example above and starting with the indirect effect (via hindsight bias), such an effect would be present if the outcome feedback first led to exaggerated impressions of the foreseeability of a fatal outcome and these in turn to negative evaluations of the decision to perform the operation and of the surgeon's competence. A direct effect of outcome knowledge (outcome bias) would be present if observers let their evaluations be influenced by the outcome irrespective of any associated foreseeability impressions, for example in an effort to restore a sense of justice by 'punishing' the surgeon for the fatal outcome (Alicke, 
2000; Tostain \& Lebreuilly, 2008). The precise nature of the reasons, justifications, or more generally processes involved in direct outcome effects depends of course on the specific context of a case, but there are two essential requirements that distinguish them from the previously discussed indirect effects: (1) that they bypass any hindsight-related cognitions and (2) that they are driven by outcome valence, not by outcome knowledge per se. The latter is perhaps taken mostly for granted in the outcome bias literature, but it is an important conceptual distinction to keep in mind.

\section{The present approach: Investigation of direct/independent and indirect/mediational effects of outcome knowledge}

Figure 1 illustrates the importance of the conceptual distinction between outcome knowledge and outcome valence. If outcome knowledge and outcome valence are conceptually conflated (as in Panel a), then hindsight bias (through hindsight perceptions of outcome foreseeability) becomes an indirect effect. This is Baron and Hershey's (1988) view of the role of hindsight bias in outcome effects (as we understand it). In our

[800]

alternative view, these aspects are conceptually separated (as in Panel b), and outcome valence - as a separate aspect of the outcome - and outcome foreseeability exert independent effects on behaviour/decision evaluation. Perhaps the clearest illustration of the difference in emphasis is in terms of our subsequent statistical analyses: While Baron and Hershey's view translates into a mediation model in which outcome knowledge/valence has direct and indirect (mediated through outcome foreseeability) effects on behaviour/decision evaluation, our alternative view lends itself to a simple regression model (i.e., behaviour/decision evaluation regressed on outcome valence and foreseeability). We do not think that either model will be true or false under all circumstances; we rather see this as an empirical question to be answered on a case-by-case basis, using the conceptual and analytical framework developed here.

In this paper, we analyse the (direct or indirect) roles of outcome valence and hindsight foreseeability impressions as explained above, in the context of people's retrospections on the London 2012 Olympic Games. To our knowledge, this is the first study with this explicit purpose - previous outcome bias research has focussed on direct outcome effects, seeking to eliminate indirect effects by design (Baron \& Hershey, 1988), whereas previous hindsight bias research was aimed at hindsight effects, but not at isolating direct outcome effects. Specifically, we investigated outcome effects in retrospective evaluations of the decision of the International Olympic Committee (IOC) to award the 2012 Olympics to London. This being a naturalistic case study, we had to rely on people's unmanipulated perceptions of outcome valence and consequently tested a correlational version of the outcome effect - that people's evaluations of the IOC decision should covary with the perceived quality of the London Olympics (i.e., better quality, better decision, and lower quality, worse decision). 
(a)

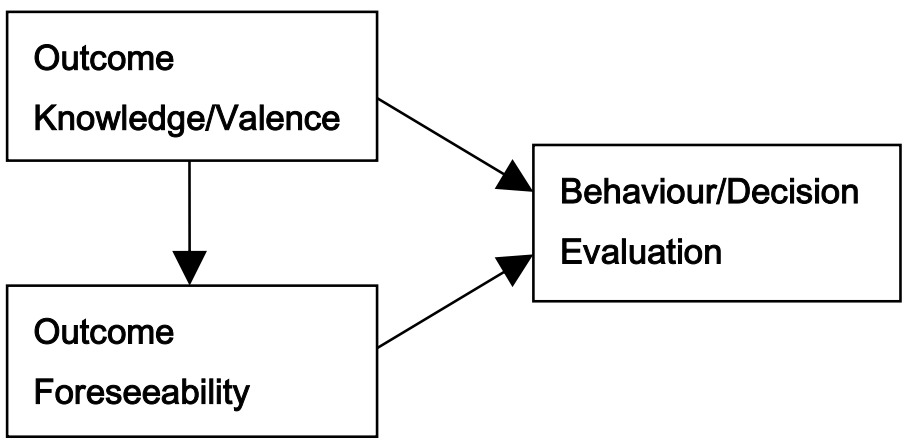

(b)

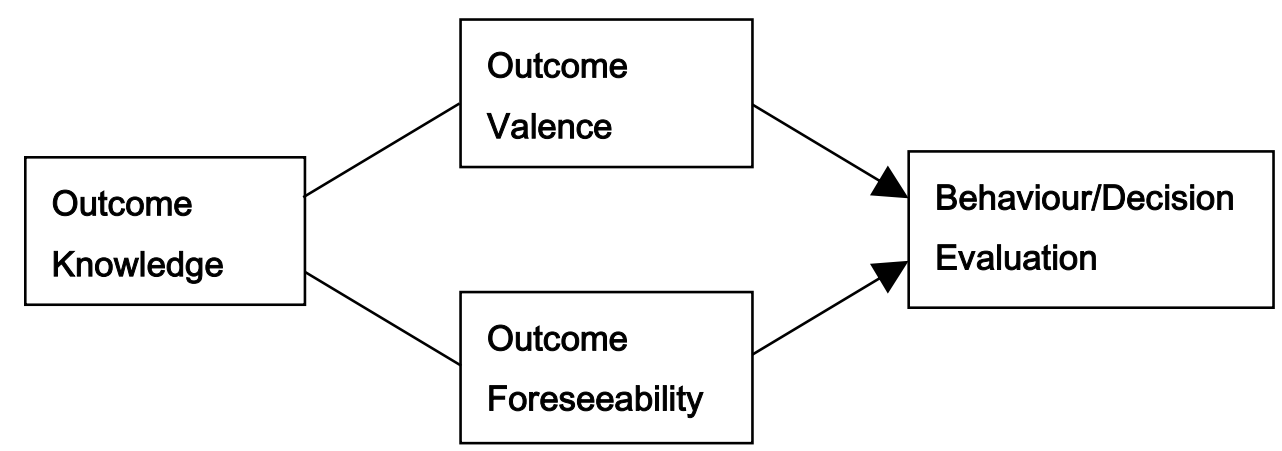

Figure 1. Two ways of thinking about the contribution of outcome and hindsight effects to behaviour/decision evaluation. Panel (a) shows a direct outcome effect and an indirect hindsight effect; panel (b) shows independent and direct outcome (valence) and hindsight (outcome foreseeability) effects.

\section{Method}

\section{Participants and recruitment}

Three hundred and sixty-seven members of the general public volunteered to take part in our online study, coming from two separate samples recruited in the United Kingdom

[800]

[801]

(through the University of Portsmouth website) and Germany (through an online studies participant pool at the University of Düsseldorf), respectively. Of these 367 participants, 282 provided sufficiently complete data sets for analysis (i.e., with no more than one missing value for the key measures, not counting the demographic data). The final UK sample consisted of 123 respondents ( 82 women and 40 men, 1 not indicated; age range: $18-65$ years, $\mathrm{Mdn}=32 ; 80 \%$ had or were currently enrolled in university education; 98\% were UK residents). The final German sample consisted of 159 respondents (98 women and 58 men, 3 not indicated; age range: $18-66$ years, $\operatorname{Mdn}=29.5 ; 76 \%$ had or were currently enrolled in university 
education; 97\% were German residents). Both samples were recruited approximately 2 weeks after the closing of the Olympics. Over $90 \%$ of the respondents completed the questionnaire within the first week after it went live.

\section{Online questionnaire}

The online questionnaire (entitled 'Looking back on the London Olympics') was programmed and designed using specialized Internet survey software (EFS survey 9; QuestBack, 2012). The start page explained that we were interested in the participant's 'views on a number of issues related to the London Olympics and the International Olympic Committee's decision to hold the games in London'. The next two pages (both entitled 'Were the London Olympics a success or a failure?') assessed participants' evaluation of the London Olympics. The first page explained that we were 'interested in your opinions on some key factors [meant to cover the most important determinants of the success of a large-scale outdoor event such as the Olympics] and in your overall evaluation'. Specifically,we asked participants to rate, on 4-point response scales with the verbal anchors Complete Failure/Very Poor (coded as 1) to Complete Success/Very Good (coded as 4), the following items (exact wordings): (1) Quality of sport events (performances, records, drama, atmosphere, fairness, doping issues, etc.), (2) Quality of organisation (venues, tickets, transport, timing of events, friendliness of support staff, security etc.), (3) Opening and closing ceremonies, (4) Finances/value for money, (5) Weather conditions, (6) Quality of media coverage (competence, entertainment value, lack of bias, etc.), and (7) Overall. The second evaluation page then asked participants to provide another overall evaluation of the London Olympics, on the same failure-success scale as before, but this time relative to the four preceding Olympics (Atlanta 1996, Sidney 2000, Athens 2004, and Beijing 2008) and - counterfactually - relative to once-possible alternative host cities (Madrid, Moscow, New York, and Paris).

The following page (entitled 'Your involvement') functioned as a buffer between the outcome valence pages and the subsequent pages covering outcome and hindsight effects, for explorative purposes, and to try and reduce possible demand characteristics by making the relation between these two parts less obvious. This page covered various aspects of the participants' involvement in the London Olympics - attendance of live events, watching TV coverage in various forms, and planning of their time around specific events (see Additional Materials for details).

The next questionnaire page (entitled 'Looking back on the IOC decision to hold the 2012 Olympics in London') first provided some historical context for the IOC decision that was the focus of our study. We explained that the final IOC decision in 2005 was between five competitor cities, following a site visit report by an IOC inspection team that slightly favoured Paris, closely followed by London. Then, we asked the participants to 'think back to the time when the decision was made and announced' and rate the quality of the decision, on a 4-point scale with the verbal anchors 'disagree' and 'agree' and using

[801]

[802]

the following exact item wordings: (1) The process leading to the 2005 IOC decision to award the Games to London was clear and transparent, (2) The IOC decision was fair and unbiased, (3) The decision was made 
responsibly, taking all relevant information into account, and (4) Overall, it was a good decision to award the 2012 Olympic Games to London.

Two further sets of items on the same page but in separate blocks, and using the same disagree-agree response format, assessed impressions of the foreseeability and inevitability of the London Olympics' outcome. ${ }^{2}$ Modelled closely after similar item sets used in previous hindsight bias studies (Blank, Nestler, von Collani, \& Fischer, 2008; Nestler, Blank, \& Egloff, 2010; Nestler \& Egloff, 2009), the foreseeability set included the following items: (1) I knew all along how the London Olympics would turn out, (2) The ultimate outcome of the London Olympics was difficult to predict (reverse-scored), (3) I had a clear and vivid idea of the outcome, and (4) Overall, the outcome of the London Olympics was foreseeable. The order of assessment of the decision evaluation and of foreseeability (and inevitability) was randomized. ${ }^{3}$ Two final questionnaire pages debriefed the participants and asked for demographic data. Screenshots of the online questionnaire pages are included as Additional Materials.

\section{Results}

Our core results are correlation/regression and mediation analyses addressing the impact of outcome valence and outcome foreseeability on the evaluations of the IOC decision to give the 2012 Olympics to London, but we start with measurement reliability analyses and a general description of how the UK and German samples looked back on the London Olympics.

\section{Measurement reliability analyses and general description}

As our approach did not involve an outcome manipulation but relied on 'natural' outcome valence, that is, people's own perceptions of the quality and success of the London Olympics, reliable measurement of this outcome valence is crucial. An 8-item measure including the seven ratings of different aspects of the Games (e.g., sport events and the weather, including the 'overall' rating), as well as the 'London' rating from the comparative assessment in the context of previous Olympics and counterfactual 2012 locations, proved satisfactory. The Cronbach's alphas for this measure were .80 for the UK sample and .81 for the German sample. The foreseeability scale was reliable as well, with Cronbach's alphas of .89 (UK) and .78 (GER). Finally, the alphas for our decision evaluation scale were satisfactory, .76 (UK) and .83 (GER).

Table 1 describes the key variables in our two samples (initial inspection revealed no extreme outliers for any of the variables, so we kept all cases for our analyses). Although comparisons between the two samples were not of key interest to us, we report these

\footnotetext{
${ }^{2}$ Inevitability was included for exploratory purposes only, and these results do not add to any of the analyses and conclusions in this paper. These questionnaire pages also contained an unsuccessful manipulation of foreseeability. Both will not be mentioned here any further but are described in the Additional Materials.

${ }^{3}$ Due to a programming error, this restriction was not followed for about a twelfth of the participants in the UK survey, meaning that for these participants the decision evaluation would have been assessed in between the foreseeability and inevitability measures. In any case, separate ANOVAs for the main dependent variables (decision evaluation and foreseeability) showed that presentation order did not have a significant impact on the overall findings, neither in the UK sample nor in the German sample.
} 
data as background information. Essentially, both samples perceived the London Olympics as a success, but the UK sample more so than the German sample - perhaps not so surprising for the hosts of the Games and also on the basis of the respective achievements of the two countries (the UK - or Team GB - ranking 3rd and Germany ranking 6th in the final medal table). Further, the UK sample valued the IOC decision to give the Games to London more highly than the German sample did; in fact, the German decision evaluation was hardly above the neutral midpoint of the scale (2.5). Further, both samples saw the Games' outcome on the unforeseeability side of the scale (with little difference between them).

\section{Main analyses}

Simple correlations established clear outcome effects in both samples. The correlations between outcome valence and the evaluation of the IOC decision were $r=.46$ (95\% CI: $0.31,0.59 ; \mathrm{p}<.001)$ in the UK sample and $r=.36$ (95\% CI: $0.21,0.49 ; p<.001)$ in the German sample. The next and crucial step, following the theoretical analysis in our introduction, was to investigate the direct or indirect contributions of outcome valence on the one hand and hindsight foreseeability impressions on the other. Before proceeding to these analyses, however, a particular difficulty - arising from the unmanipulated nature of outcome valence in our study - had to be resolved. Depending on how respondents rated the outcome of the London Olympics (positively or negatively), foreseeability of the outcome could translate into a positive or a negative decision evaluation (i.e., there is no uniform relation to be expected). To solve this problem, we recoded (general) foreseeability into foreseeability of a positive outcome by reversing the foreseeability scores for respondents with negative outcome valence (i.e., a foreseeable negative outcome translates into an unforeseeable positive outcome). As seven respondents were exactly neutral about the outcome (valence of 2.5), they were excluded from further analysis, reducing the samples sizes for the subsequent analyses marginally to Ns of 122 (UK) and 153 (GER).

After this recoding, we conducted a simple mediation analysis based on ordinary least squares path analysis (Hayes, 2013), using a specialized package (Tingley, Yamamoto, Hirose, Keele, \& Imai, 2014) for the computer software R (R Core Team, 2015). This approach is a modern version of Baron and Kenny's (1986) regression approach to mediation and involves (1) testing the joint direct effects of the predictor (outcome valence in our case) and of the presumed mediator (foreseeability of a positive outcome) on the dependent variable (decision evaluation), as well as (2) testing the indirect effect

Table 1. Means and 95\% Confidence Intervals for the Key Variables of Interest

\begin{tabular}{lcccccc}
\hline & \multicolumn{2}{c}{ UK sample $(N=123)$} & & \multicolumn{2}{c}{ German sample $(N=159)$} \\
\cline { 2 - 3 } \cline { 5 - 6 } Variable & $M$ & $95 \%$ CI & & $M$ & $95 \%$ CI \\
\hline Outcome valence & 3.42 & $(3.35,3.49)$ & & 3.15 & $(3.08,3.21)$ \\
Decision evaluation & 3.09 & $(2.98,3.19)$ & & 2.60 & $(2.50,2.71)$ \\
Foreseeability & 2.22 & $(2.08,2.36)$ & & 2.14 & $(2.03,2.25)$ \\
\hline
\end{tabular}


(path) of the predictor through the mediator. The resulting (unstandardized) regression/path coefficients are shown in Figure 2 (see the Additional Materials for a full table of regression coefficients) and are easily summarized: In both samples, there were significant direct effects of both outcome valence and foreseeability on decision evaluation, but the impact of outcome valence on the perceived quality of the IOC decision was stronger (i.e., there were stronger outcome than hindsight effects - the c' and b regression coefficients can be compared directly, as the predictor and the mediator were measured on the same 1-4 scales). By contrast, the indirect, mediated effect (ab) of outcome evaluation through foreseeability was estimated to be small and non-significant in both samples (UK: $a b=0.04$; bootstrapped 95\% CI: $-0.01,0.14$; $\mathrm{p}=.15 /$ GER: $\mathrm{ab}=0.03$; bootstrapped $95 \% \mathrm{CI}:-0.04,0.13 ; \mathrm{p}=.43$; bootstrapped intervals based on 100,000 samples each).

To summarize our mediation analysis, we found no evidence for an indirect, mediated influence of outcome valence through foreseeability impressions in our mediation analyses. Instead, our analyses support the idea introduced earlier (Figure 1b) that outcome valence and foreseeability are separate aspects of outcome knowledge and can exert independent, direct effects on decision evaluation. Moreover, the fact that this same principle result was obtained in samples from two different countries (with a different degree of involvement in the London Olympics; see Additional Materials) adds to the generality of our findings. 


\section{(a) UK sample $(N=122)$}

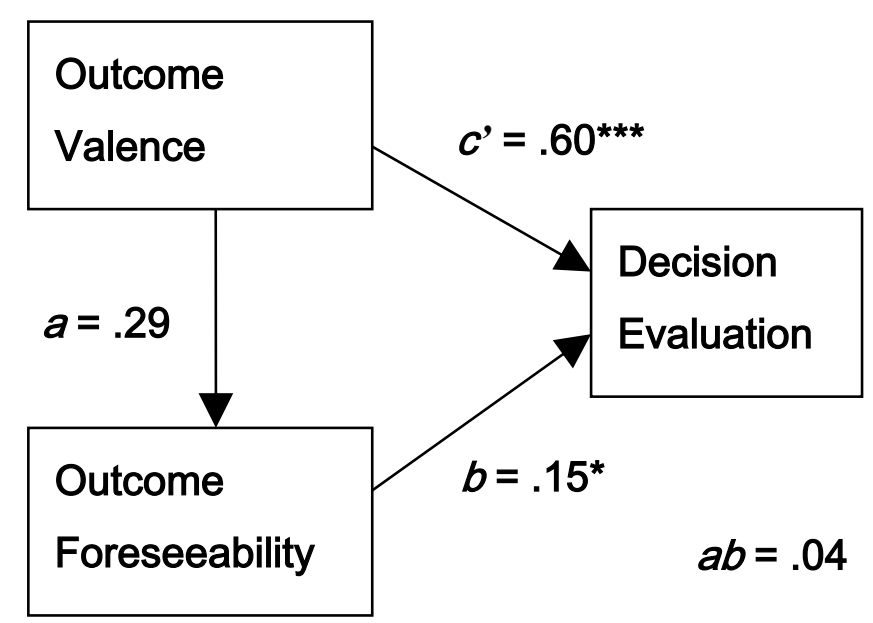

(b) German sample $(N=153)$

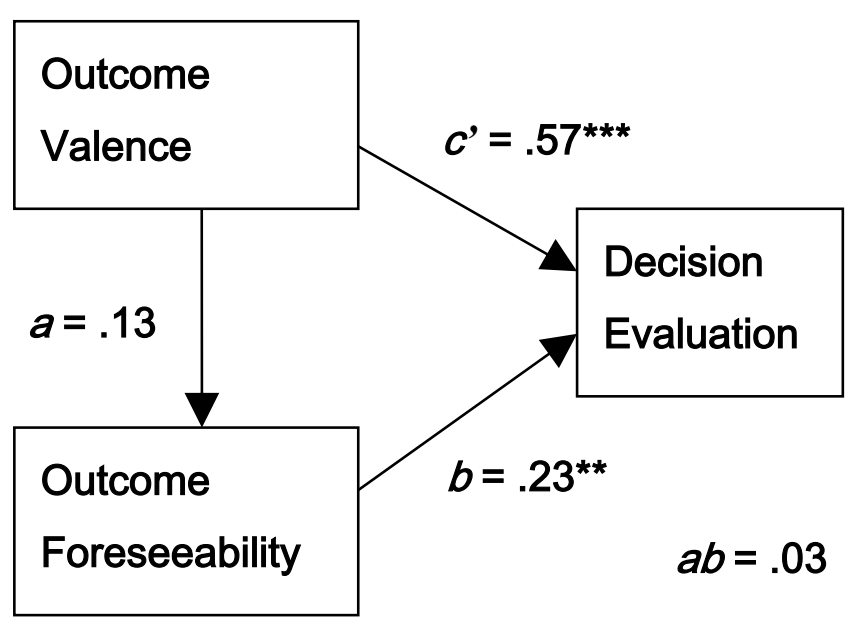

Figure 2. Simple mediation model to analyse the indirect effect of outcome valence on decision evaluation through outcome foreseeability (ab). $* \mathrm{p}<.05 ; * * \mathrm{p}<.01 ; * * * \mathrm{p}<.001$.

[804]

[805]

\section{Discussion}

The main message of our study with respect to the relation between outcome bias/effects and hindsight bias/effects is that both can make independent contributions to the evaluation of decisions. Specifically, our respondents' evaluations of the IOC decision to choose London as the host of the 2012 Olympic Games depended both on how big a success they felt the Games had been and on how foreseeable a positive outcome was for them. The fact that the outcome effect was not mediated through foreseeability reinforces 
our idea that outcome valence and outcome foreseeability are separate consequences of outcome knowledge and operate largely independent of each other (Figure 1b). We do not necessarily take these findings to positively contradict the idea of indirect (via foreseeability) outcome effects (Baron \& Hershey, 1988), however. Rather, which model 'prevails' may depend on circumstances; in any case, this is an empirical question that can be addressed in future joint investigations of outcome and hindsight effects. The present contribution provides a conceptual framework for such investigations. For even greater conceptual clarity, we suggest to speak in the future of outcome valence effects, not just outcome effects, to highlight that not outcome knowledge per se, but people's evaluation of outcomes is the essential ingredient in these effects. We believe these conceptual contributions are just as interesting and important as our empirical findings; research always progresses as a reciprocal interaction between these two elements. Along similar lines, we would like to conclude by briefly commenting on another conceptual issue related to the normative interpretation of outcome effects, specifically, whether or not it is justified to speak of a bias here. We note (agreeing with observations by other authors, Baron \& Hershey, 1988) that to some degree outcome 'bias' is built into our colloquial language. It is quite natural to say, for instance, that it was 'a good decision to have the fish' at a restaurant dinner if it turned out to be delicious, and the same sort of language would be entirely appropriate in many other contexts. In this sense, it does not make sense to picture people, from a narrow scientific point of view, as being outcome-biased. On the other hand, we of course also agree that there are important classes of situations in which speaking of a bias is entirely justified, that is, where blaming or praising decision-makers for decisions with particular outcomes would not be appropriate (see Baron \& Hershey, 1988; Hershey \& Baron, 1992, 1995, for extensive discussions).

The root of the problem, we believe, is that the term 'decision' is ambiguous - it can refer to either the decision-as-it-happened, an event that includes the decision outcome, or the process of making the decision, which does not include the outcome. Both understandings are covered by the term 'decision', and therefore part of the bias question is which meaning people have in mind when they evaluate a 'decision'. As many studies of outcome bias have simply asked their participants to evaluate 'the decision' (or similar), it is therefore not fully clear what they have actually measured.

There is some empirical support for this conceptual analysis from our own study. Looking at the items we used to measure 'decision' evaluation (for convenience repeated here: [1] The process leading to the 2005 IOC decision to award the Games to London was clear and transparent, [2] The IOC decision was fair and unbiased, [3] The decision was made responsibly, taking all relevant information into account, and [4] Overall, it was a good decision to award the 2012 Olympic Games to London), the first three would seem to pertain to the decision-making process, whereas the fourth, summary item would seem to reflect the global decision event. Confirming this impression, an explorative factor analysis enforcing a 2 -factor solution (although a 1-

[805]

[806]

factor solution was satisfactory as well) and using varimax rotation found, in both the UK and German samples, that the first three items were clearly grouped on a first factor and the summary item on a second 
(rotated factor loadings $>0.8$ on the relevant factor and $<0.4$ on the other in both samples). Hence, it does seem to be important, from a normative perspective, exactly how 'decision' evaluation is measured. If this idea had not occurred to us only in hindsight, we would have paid more attention to this particular measurement problem from the outset, but in any case we think this is a worthwhile issue to address in future research.

\section{Acknowledgement}

We wish to thank Julia Gro $\beta$ for helpful comments on a draft of this paper.

\section{References}

Alicke, M. D. (2000). Culpable control and the psychology of blame. Psychological Bulletin, 126, 556-574. doi:10.1037/0033-2909.126.4.556

Baron, J., \& Hershey, J. C. (1988). Outcome bias in decision evaluation. Journal of Personality and Social Psychology, 54, 569-579. doi:10.1037/0022-3514.54.4.569

Baron, R. M., \& Kenny, D. A. (1986). The moderator-mediator variable distinction in social psychological research: Conceptual, strategic, and statistical considerations. Journal of Personality and Social Psychology, 51, 11731182. doi:10.1037/0022-3514.51.6.1173

Blank, H., Musch, J., \& Pohl, R. F. (2007). Hindsight bias: On being wise after the event. Social Cognition, 25, 1-9. doi:10.1521/soco.2007.25.1.1

Blank, H., Nestler, S., von Collani, G., \& Fischer, V. (2008). How many hindsight biases are there? Cognition, 106, 1408-1440. doi:10.1016/j.cognition.2007.07.007

Brown, C. E., \& Solomon, I. (1987). Effects of outcome information on evaluations of managerial decisions. The Accounting Review, 62, 564-577.

Clarkson, P. M., Emby, C., \& Watt, V. W.-S. (2002). Debiasing the outcome effect: The role of instructions in an audit litigation setting. Auditing: A Journal of Practice \& Theory, 21, 7-20. doi:10.2308/aud.2002.21.2.7

Fischhoff, B. (1975). Hindsight 61/4 foresight: The effect of outcome knowledge on judgment under uncertainty. Journal of Experimental Psychology: Human Perception and Performance, 1, 288-299. doi:10.1037/00961523.1.3.288

Gupta, M., Schriger, D. L., \& Tabas, J. A. (2011). The presence of outcome bias in emergency physician retrospective judgments of the quality of care. Annals of Emergency Medicine, 57, 323-328.e9. doi:10.1016/j.annemergmed.2010.10.004

Hawkins, S. A., \& Hastie, R. (1990). Hindsight: Biased judgments of past events after the outcomes are known. Psychological Bulletin, 107, 311-327. doi:10.1037/0033-2909.107.3.311

Hayes, A. F. (2013). Introduction to mediation, moderation, and conditional process analysis: A regression-based approach. New York, NY: Guilford Press.

Hershey, J. C., \& Baron, J. (1992). Judgment by outcomes: When is it justified? Organizational Behavior and Human Decision Processes, 53, 89-93. doi:10.1016/0749-5978(92)90056-D

Hershey, J. C., \& Baron, J. (1995). Judgment by outcomes: When is it warranted? Organizational Behavior and Human Decision Processes, 62, 127. doi:10.1006/obhd.1995.1038

Hoffrage, U., \& Pohl, R. (2003). Research on hindsight bias: A rich past, a productive present, and a challenging future. Memory, 11, 329-335. doi:10.1080/09658210344000080

Jones, S. K., Yurak, T. J., \& Frisch, D. (1997). The effect of outcome information on the evaluation and recall of individuals' own decisions. Organizational Behavior and Human Decision Processes, 71, 95-120. doi:10.1006/obhd.1997.2714 
Lipshitz, R., \& Barak, D. (1995). Hindsight wisdom: Outcome knowledge and the evaluation of decisions. Acta Psychologica, 88, 105-125. doi:10.1016/0001-6918(95)94011-X

Mazzocco, P. J., Alicke, D.,\&Davis, T. L. (2004).Onthe robustness of outcome bias: No constraint by prior culpability. Basic and Applied Social Psychology, 26, 131-146. doi:10.1080/01973533.2004.9646401

Mowen, J. C., \& Stone, T. H. (1992). An empirical analysis of outcome biases in constituent evaluations of public policy decision makers. Journal of Public Policy \& Marketing, 11, 24-32.

Nestler, S., Blank, H., \& Egloff, B. (2010). Hindsight 61/4 hindsight: Experimentally induced dissociations between hindsight components. Journal of Experimental Psychology: Learning, Memory, and Cognition, 36, 13991413. doi:10.1037/a0020449

Nestler, S., \& Egloff, B. (2009). Increased or reversed? The effect of surprise on hindsight bias depends on the hindsight component. Journal of Experimental Psychology: Learning, Memory, and Cognition, 35, $1539-1544$. doi:10.1037/a0017006

QuestBack. (2012). Unipark EFS Survey 9.0. Retrieved from http://www.unipark.de

R Core Team. (2015). R: A language and environment for statistical computing. Vienna, Austria. Retrieved from http://www.R-project.org

Rachlinski, J. J. (1998). A positive psychological theory of judging in hindsight. The University of Chicago Law Review, 65, 571-625. doi:10.2307/1600229

Robbennolt, J. K. (2000). Outcome severity and judgments of "responsibility": A meta-analytic review. Journal of Applied Social Psychology, 30, 2575-2609. doi:10.1111/j.1559-1816.2000.tb02451.x

Roese, N. J., \& Vohs, K. D. (2012). Hindsight bias. Perspectives on Psychological Science, 7, 411-426. doi:10.1177/1745691612454303

Tingley, D., Yamamoto, T., Hirose, K., Keele, L., \& Imai, K. (2014). Mediation: R package for causal mediation analysis. Journal of Statistical Software, 59, 1-38. Retrieved from http://www.jstatsoft.org/v59/i05/

Tostain, M., \& Lebreuilly, J. (2008). Rational model and justification model in 'outcome bias'. European Journal of Social Psychology, 38, 272-279. doi:10.1002/ejsp.404

Received 10 September 2014; revised version received 8 April 2015

\section{Supporting Information}

The following supporting information may be found in the online edition of the article:

Data S1. Supporting information. 